\title{
Indexing Kiez - Zur Deethnisierung juventulektaler Stile
}

\author{
Indexing Kiez - Deethnization of Adolescent Speechstyles
}

\begin{abstract}
ZUSAMMENFASSUNG ${ }^{1}$
Monolinguale Sprecher*innen wurden in der Vergangenheit eher weniger mit ethnolektalem Sprechen in Verbindung gebracht. Verschiedene Korpora zeigen jedoch zunehmend Elemente, die dem Kiez- oder Türkendeutsch zugeordnet wurden - auch in monolingualen Daten. In unserem Beitrag zeigen wir an drei Beispielen prominente Merkmale ethnolektaler Sprechweisen, die jedoch die außersprachliche Dimension Ethnie nicht mehr direkt spiegeln. Wir plädieren abschließend für eine Verortung der Daten im Stilspektrum Jugendlicher, die Straßentauglichkeit und Härte der Sprecher*innen indizieren sollen.

Schlüsselwörter: Deethnisierung, Kiezdeutsch, Indizierung von Straßentauglichkeit, Jugendsprache
\end{abstract}

\section{ABSTRACT}

Monolingual speakers have in the past been less associated with ethnolectal speaking. However, various corpora increasingly show elements that were assigned to Kiez- or Turkic-German - even in monolingual data. In our article we show prominent features of ethnolectal speechstyles by giving three examples, which no longer directly reflect the extra-linguistic dimension ethnicity. In conclusion, we advocate locating the data in the stylespectrum of adolescents, which should indicate a streetcredibility and toughness of the speakers

Keywords: deethnization, Kiezdeutsch, indexing street, jouth language

\section{Einleitung}

Die wissenschaftliche Betrachtung multilingualer Lebenswelten als Teil einer Kultur hat - gehen wir im deutschen Sprachraum weitschweifend von Grimm (1846, zit. nach 1986) aus - eine über hundertfünfzigjährige Tradition. Erst seit Mitte der 1990er Jahre sind jedoch Spielarten des Sprechens Jugendlicher ein Teil der migrationsbedingten Diskussionen über Sprache geworden. Frühe Arbeiten der

1 Für hilfreiche Kommentare, Verbesserungen und Ideen danken wir herzlich Stefanie Krain (Münster), Norbert Dittmar (Berlin) und den beiden Gutachter*innen, die wertvolle Hinweise zur Optimierung des Textes gegeben haben.

Nils Bahlo, Germanistisches Institut, Westfälische Wilhelms-Universität Münster, Schlossplatz 34, 48143 Münster, Phone: 004902518324657, n.bahlo@uni-muenster.de, https://orcid.org/0000-00019769-0607

Sharon Lohse, Germanistisches Institut, Westfälische Wilhelms-Universität Münster, Schlossplatz 34, 48143 Münster, s.lohse@uni-muenster.de, https://orcid.org/0000-0001-6409-1050 
deutschen Linguistik besprechen vor allem die Formen ethnolektaler Sprechweisen Jugendlicher (u.a. Füglein, 2000). Später treten auch funktionale Aspekte in den Fokus. Auer (2003, auch leicht revidiert 2016) zeigt mit seinem Modell, dass diese Sprechweisen keineswegs isoliert stehen, sie werden von Jugendlichen ohne Migrationshintergrund - teils direkt, teils indirekt (z.B. über Medien) - rezipiert und gegebenenfalls sogar adaptiert. Erstmalig konnten in einer Studie von Dirim und Auer (2004) Anzeichen von Deethnisierungsprozessen festgestellt werden. Weitere Untersuchungen blieben jedoch in der deutschsprachigen Linguistik weitestgehend aus (vgl. dazu Artamonova, 2016). Wiese (u.a. 2012) betont erstmalig durch den Begriff „Kiezdeutsch“ die diatopische Varietätendimension multiethnolektaler Stile. Es scheint, als rücke die Ethnie zunehmend in den Hintergrund der außersprachlichen Dimension jugendlicher Stile, die vormals Jugendlichen mit Migrationshintergrund zugeschrieben wurden.

An Berliner Daten, die von Jugendlichen ohne Migrationshintergrund stammen, wollen wir gesprächsanalytisch herausarbeiten, welche Bestandteile des (unter anderem) „Kiezdeutsch“ genannten in Sprechstile Jugendlicher ohne Migrationshintergrund Einzug gehalten haben. Auf der Folie unserer Daten zeigen wir, welche außersprachlichen Dimensionen abseits der Ethnie die Sprecher*innen durch ihren Sprachgebrauch indizieren. $\mathrm{Zu}$ ihnen zählen u.a. die diatopische Dimension „Kiez“ und eine damit verbundene diastratische (milieuspezifische) Variation im Sinne eines „Indexing Street“ (Steckbauer, Bahlo, Dittmar, \& Pompino-Marschall, 2014).

\section{Ethnolektsysteme und Identiät}

Das Verhältnis von Sprache und Identität ist vielfach Thema wissenschaftlicher Betrachtungen gewesen. Der Bezug von Sprache und Identität kann dabei mit sehr verschiedenen Ansätzen und Bezügen angegangen werden. Einer dieser Ansätze kennzeichnet sich dadurch, dass Sprache als „Medium der Selbstdarstellung“ und somit als „Präsentation der eigenen Identität“ betrachtet wird (Thim-Mabrey, 2003, S. 3). Keupp (2012) konkretisiert den Identitätsbegriff durch sein Konzept des „Patchworkings“:

Identitätsarbeit hat als Bedingung und als Ziel die Schaffung von Lebenskohärenz. In früheren gesellschaftlichen Epochen war die Bereitschaft zur Übernahme vorgefertigter Identitätspakete das zentrale Kriterium für Lebensbewältigung. Heute kommt es auf die individuelle Passungsund Identitätsarbeit an, also auf die Fähigkeit zur Selbstorganisation, zum ,Selbsttätigwerden ‘ oder zur ,Selbsteinbettung'. Das Gelingen dieser Identitätsarbeit bemisst sich für das Subjekt von innen an dem Kriterium der Authentizität und von außen am Kriterium der Anerkennung [Hervorhebung original] (S. 9).

Das bedeutet demzufolge, dass Identität durch Sprache in situ konstituiert, ratifiziert und auch ausprobiert und ggf. gefestigt oder verworfen wird. Identitäten 
können Aspekte der Ethnie, des Glaubens, des Lebensstils, des Geschlechts, des Charakters usw. beinhalten. Besonders unter dem Gesichtspunkt von Migrationsund Mehrsprachigkeitserfahrungen werden und wurden O-Tonaufnahmen oftmals gesprächsanalytisch betrachtet. Vielen dieser Studien ist gemein, dass sie Merkmale des Sprechens Jugendlicher mit verschiedenen außersprachlichen Dimensionen in Zusammenhang bringen und nicht selten die Ethnie in den Fokus rücken.

Ein zweites Aufgabenfeld im Bereich der ethnolektalen Sprachforschung betrifft medial vermittelte Bilder eines sekundären, stilisierten Ethnolekts, der oftmals als „Kanak Sprak“ (Zaimoglu, 2011) betitelt wurde. Mit der Realität hat dieser Mediolekt, der sich durch falsche Kasusmarkierungen, fehlende Präpositionen und Artikel en gros auszeichnet, jedoch nur partiell etwas gemein. Wir folgen Auers (2003) Modell, wenn wir feststellen, dass diese stereotypen Bilder, die in der Regel Jugendlichen mit Migrationshintergrund zugeschrieben werden, über die Medienformate kolportiert werden und teilweise Einzug in den Sprachgebrauch Jugendlicher auch ohne Migrationshintergrund - im Rahmen eines tertiären Ethnolekts - finden. Androutsopoulos (2001) hat diesen Vorgang bereits vor 20 Jahren mit den Worten „from the streets to the screen and back again" treffend im Titel einer Arbeit beschrieben.

Letztendlich - und dies mag besonders in den vielfach in der Literatur beschriebenen Großstädten der Normalfall sein - führt der direkte Kontakt von Jugendlichen mit Migrationshintergrund und markant ausgeprägten ethnolektal markierten Sprechweisen zur (partiellen) Übernahme einiger dieser Merkmale durch Jugendliche ohne Migrationshintergrund. Die Gründe dafür sind unterschiedlich. Mehr oder weniger bewusste Solidarisierungshandlungen, aber auch unbewusste Stilisierungen und annähernd habitualisierte Verwendungsweisen können beobachtet werden (Artamonova, 2016; Auer \& Dirim, 1999; Bahlo et al., 2019).

Von einer Homogenität dieser drei Ethnolektsysteme auszugehen, war niemals Gegenstand linguistischer Überlegungen, sehr wohl aber die Frage nach den Unterscheidungskriterien, die wohl Gemeinsamkeiten, aber auch Unterschiede aufweisen. Trennscharfe Systemgrenzen dieser Stile bzw. Stilisierungen können wohl nicht angenommen werden. Wenn es Gemeinsamkeiten innerhalb ursprünglich ethnolektal zugeschriebener Merkmale des Sprachgebrauchs gibt, die auf dem Wege sind, über den tertiären Ethnolekt bzw. den indirekten oder auch direkten Kontakt deethnisiert $\mathrm{zu}$ werden, müssen sich frequente Beispiele innerhalb eines Korpus monolingualer deutscher Jugendlicher (ohne Migrationshintergrund) finden lassen.

\section{Methode}

Die Etablierung der Konversationsanalyse als eigenständige Forschungsrichtung geht auf die 60er Jahre der amerikanischen Soziologie zurück. Sie hat sich aus der Ethnomethodologie Harold Garfinkels (1967) entwickelt und untersucht, wie 
soziale Wirklichkeit in der Interaktion durch die Gesprächsteilnehmenden konstituiert wird. Als linguistische Disziplin fokussiert sie insbesondere die Analyse der sprachlichen Mittel, Prinzipien und kommunikativen Praktiken, anhand derer ein Gespräch gesteuert wird (vgl. u.a. Birkner, Auer, Bauer, \& Kotthoff, 2020; Deppermann, 2008).

UntersuchungsgegenstandderGesprächsanalysesindAusschnitteausschließlich natürlicher Interaktionen. Dabei geht es nicht um eine quantitative Auswertung der verwendeten sprachlichen Mittel, sondern um eine detaillierte Analyse des aufeinander aufbauenden Verlaufs. Die Grundannahme der Gesprächsanalyse besteht darin, dass dieser Verlauf in situ interaktiv ausgehandelt wird (vgl. Selting \& Couper-Kuhlen, 2001, S. 258). So wird durch eine bestimmte Reaktion immer auch deutlich, wie die vorhergehende Äußerung interpretiert wurde. Anhand dieser Reaktion kann ein*e Sprecher*in überprüfen, ob das Gegenüber die Äußerung richtig verstanden hat, und gegebenenfalls eine Reparatur einleiten (vgl. Birkner et al., 2020, S. 239). Nach Deppermann (2008) ist die detaillierte Sequenzanalyse deshalb das „Herzstück der Gesprächsanalyse“ (S. 53). Dabei werden (non-) verbale Äußerungen im sequenziellen Kontext betrachtet, das heißt, immer zu der vorigen und folgenden Handlung in Bezug gesetzt. Das bedeutet gleichzeitig, dass keine Äußerungen ausgelassen werden dürfen. Dieses Vorgehen ist notwendig, um während der Analyse auf einer Höhe mit den Interaktionsteilnehmer*innen zu bleiben und den Gesprächsverlauf nicht anhand von Äußerungen zu erklären, die erst im späteren Verlauf gemacht werden (vgl. S. 54).

Im Zentrum gesprächsanalytischer Untersuchungen stehen die drei Fragen nach der Handlung (Welche Handlungen werden wie vollzogen?), Ordnungsstruktur (Welche Regularitäten bestehen hinsichtlich des sozialen Handelns und wie ist dies strukturiert?) und Intersubjektivität (Wie wird wechselseitiges Verstehen in der Interaktion konstituiert?) (vgl. Birkner et al., 2020, S. 19). Das Ziel ist also die Rekonstruktion der Verfahren, durch die die Interaktionspartner*innen den Gesprächsverlauf organisieren und Sinn herstellen bzw. gemeinsame und individuelle Zwecke verfolgen. Bei diesen Praktiken handelt es sich um (Ethno-)Methoden, die der Konstruktion, Interpretation und Organisation von Beiträgen dienen. In diesem Zusammenhang spielt das „recipient design“, also der adressatenspezifische Zuschnitt einer Äußerung, eine wichtige Rolle (vgl. Sacks, Schegloff, \& Jefferson, 1974, S. 727), denn die Ethnomethoden müssen für das Gegenüber verständlich sein. Meist handelt es sich also um kulturell mehr oder weniger verbreitete, routinierte Praktiken (vgl. Bahlo et al., 2019, S. 197).

Das Vorgehen einer Gesprächsanalyse ist rein induktiv. Das bedeutet, dass keine zuvor aufgestellten Hypothesen überprüft werden sollen. Vielmehr werden die gesprächssteuernden Ordnungsstrukturen durch die beschriebene Sequenzanalyse aufgedeckt. Die analysierten Ergebnisse müssen aus den Daten abgelesen und 
gleichzeitig durch sie bestätigt werden, Spekulationen sind dementsprechend unzulässig (Bahlo et al., 2019, S. 198). Anfangs wurde in der Gesprächsanalyse hauptsächlich mit Audioaufnahmen gearbeitet, neuere Untersuchungen ziehen aber auch Videoaufnahmen hinzu, sodass auch para- und nonverbale Mimik, Gestik usw. Bestandteil der Untersuchungen sind.

\section{Empirischer Untersuchungskontext}

Die Daten der Untersuchung stammen aus dem von der Deutschen Forschungsgemeinschaft (DFG) geförderten Projekts „Jugendsprache im Längsschnitt, DI279/16“ unter der Leitung von Norbert Dittmar. Es handelt sich um Audioaufnahmen, die 2005 bis 2010 in einem bayerischen Zeltlager einer Berliner Jugendorganisation erhoben wurden.

Die Kerngruppe der Proband*innen besteht aus drei Mädchen und drei Jungen, die schon vor den ersten Aufnahmen in das Zeltlager gefahren waren. Die Mitglieder dieser Kerngruppe bilden eine „Community of Practice“ (Eckert \& McConnelGinet, 1992, S. 461): Sie besuchen dieselbe Schule, stammen aus ähnlichen Verhältnissen und verbringen gemeinsam ihre Freizeit. Laut Eigenaussagen haben die Gruppenmitglieder nur wenig Kontakt zu Jugendlichen mit Migrationshintergrund. $\mathrm{Zu}$ Beginn der Aufnahmen sind sie 14 Jahre alt. Sowohl die Jugendlichen als auch ihre Eltern willigten den Sprachaufnahmen und ihrer Veröffentlichung ein.

Um möglichst natürliche Daten zu erhalten, wurden in den Zelten Miniaturmikrofone mit kleinen Vorverstärken versteckt angebracht. Die Aufnahmen liefen nicht den ganzen Tag, sondern wurden meistens mit Beginn der Nachtruhe um 22 Uhr gestartet, sodass sich die Jugendlichen getrenntgeschlechtlich in ihren Zelten befanden. Die Gespräche waren sehr vielfältig. Es wurden unter anderem Geschehnisse des Tages rekapituliert, Liebschaften und sexuelle Fantasien diskutiert, Betreuer und Nachbarzelte provoziert, um die Nachtruhe zu umgehen, usw.

Insgesamt kamen durch die Aufnahmen über 150 Stunden an Audiomaterial zusammen. Das Korpus wurde in einem Transkriptband (Bahlo \& Fladrich, 2016) für Forschungszwecke aufgearbeitet, nach dem Gesprächsanalytischen Transkriptionssystem (GAT2) (Selting et al., 2009) transkribiert und zugängig gemacht. In diesem Beitrag können nur exemplarisch einzelne Gesprächsausschnitte verwendet werden. Diese wurden jedoch so gewählt, dass sie in Bezug auf unsere Forschungsfrage einen weitgehend repräsentativen Eindruck vermitteln. Mittels eines kleinen Datensets sollte es möglich sein, erste Eindrücke zu gewinnen und Schlussfolgerungen für nachfolgende Arbeiten zu ziehen.

\section{Analyse}

Unser erstes Beispiel (1) stammt aus einem Mädchenzelt zur Nachtruhe nach 22 Uhr. Es steht stellvertretend für viele andere Fälle, in denen der „Spaß“ der 
gemeinsamen Interaktion im Zentrum der Handlung steht (vgl. Deppermann \& Schmidt, 2001a). In unserem Ausschnitt albern die Mädchen viel herum. Katharina (Kat) singt mehrmals die Anfangszeilen des „Biene Maja“-Lieds, was dann von Sanja (San) rülpsend ergänzt wird. Die Mädchen lachen viel über die rülpsend gesprochenen Sequenzen von Sanja und fordern sie immer wieder auf, es zu wiederholen. Bereits im Vorfeld unseres partizipativen Spektakels haben die Mädchen sehr expressiv den Abend innerhalb ihres Zeltes gestaltet.

(1) (aus Bahlo \& Fladrich, 2016, S. 19)

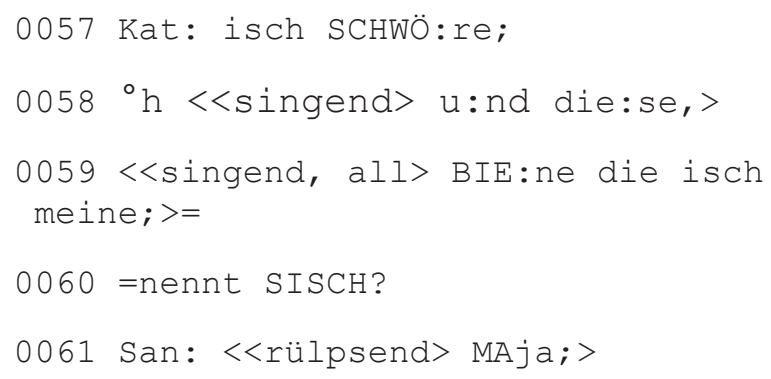

Unser Beispiel, welches aus einem größeren Transkript genommen wurde, beginnt mit der Konstruktion „ich schwöre“ (0057) (vgl. Bahlo, 2009). Auffällig an dieser Routineformel (Androutsopoulos, 1998, S. 40) ist, dass dem ,ich schwöre“" kein direktes Objekt oder ein durch den Subjunktor „dass“ eingeleiteter Komplementsatz folgt. Ein Matrixsatz mit uneingeleitetem Nebensatz liegt ebenfalls nicht vor. Auch eine Präposition wie in „Ich schwöre auf die neue Faltencreme“ wird nicht realisiert. Die Routineformel „ich schwöre“ steht syntaktisch isoliert, sie fungiert metapragmatisch als Diskursmarker und beendet die vorangegangene Sequenz, die bekräftigend und anerkennend abgeschlossen wird. Markiert erscheint in Hinblick auf andere Transkripte, in denen Katharina spricht, die Verschiebung des Artikulationsortes im ,ich“, vom /iç/ zu ,isch“/ij/. Analog dazu verfährt Katharina auch in 0059 und 0060.

Während ,ich-schwöre-Konstruktionen“ auch in monolingualen Korpora auftreten, ist hier durch die Koronalisierung eher die Nähe zu multilingualen Sprecher*innen zu sehen: Die Koronalisierung palataler Frikative wurde bislang in der Jugendsprachforschung fast durchgängig mit ethnolektalem Sprechen in Verbindung gebracht. Bekannt sind die Koronalisierungen jedoch bereits seit der zweiten Hälfte des 19. Jahrhunderts. Sie entstanden in den mitteldeutschen Großstädten und haben sich von da aus sehr schnell in den mitteldeutschen Dialekten verbreitet. Sehr konservative mitteldeutsche Dialekte haben bis heute den palatalen Frikativ (den sog. ich-Laut) bewahrt, während der koronalisierte 
Laut heute in den mitteldeutschen Dialekten und Umgangssprachen dominiert. Ein externer Grund für die Entstehung in den normtoleranten Großstädten war der Sprachkontakt mit Sprecherinnen, die den Laut nicht kannten (vgl. Herrgen, 1986, S. 226).

In diesem Beispiel fungiert die Verschiebung des Artikulationsortes jedoch nicht als ethnischer oder dialektaler Marker. Der stilistische Bruch zur „,normalerweise“ normkonformen Aussprache der Berliner Mädchen, die fast durchgängig ohne dialektale oder ethnolektale Markierungen auskommt, zeigt hier einen Kontrast zum alltagstauglichen und eher prestigeorientierten Sprachgebrauch der Probandinnen. Der abweichende Sprachgebrauch rahmt eine normabweichende tabuisierte Handlung (Lieder singen und laut in der Teilöffentlichkeit des schalldurchlässigen Zeltes rülpsen). Die Gruppe konstruiert sich ex negativo und bringt dies inhaltlich aber auch stilistisch öffentlich wirksam hervor. Indiziert wird eine „Straßentauglichkeit“, die sich eher an den stigmatisierten Verhaltensweisen orientiert, die stereotyp jungen Männern zugeschrieben wird. Der ursprünglich ethnische Marker der Koronalisierung erfährt hier offenbar eine Ausbleichung zugunsten einer „krassen“ Selbstdarstellungsmöglichkeit und der Soldarisierung im Inneren des Freundinnenkreises: Sowohl das Rülpsen in der Öffentlichkeit als auch der sprachliche Stilbruch markieren das gemeinsame Tragen der Situation in der Interaktion. Auer und Hinskens (2005, S. 336) zeigen im Rahmen ihrer Arbeit über die interpersonellen Gründe des Sprachwandels, dass es sich bei diesen kurzen Übernahmen um die hierarchisch niedrigste Stufe des Sprachwandels handelt, da lediglich episodenhaft markierte Formen eingebettet werden.

Unser zweites Beispiel (2) zeigt einen Ausschnitt aus bzw. vor einem Jungenzelt. Die Jugendlichen sollen die Nachtruhe einhalten und die Lautstärke senken. Da die gewünschte Verhaltensweise jedoch nicht mit ihren Vorstellungen übereinstimmt, versuchen sie im folgenden Ausschnitt, den Betreuer Simon durch verbale Attacken im Rahmen eines „Battles“ zu provozieren. Die Strategie geht auf, das gegenseitige „Dissen“ (Deppermann \& Schmidt, 2001b) zögert die Nachtruhe 10 Minuten hinaus.

(2) (aus Bahlo \& Fladrich, 2016, S. 23)

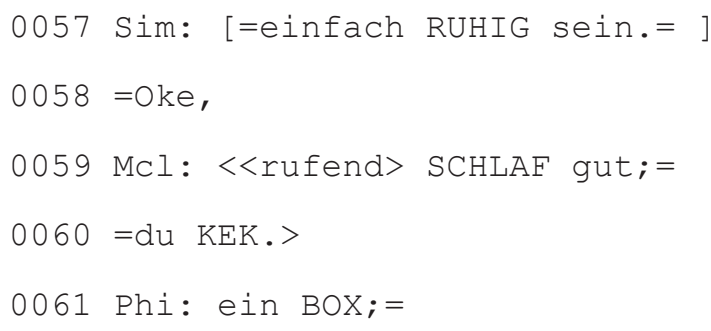




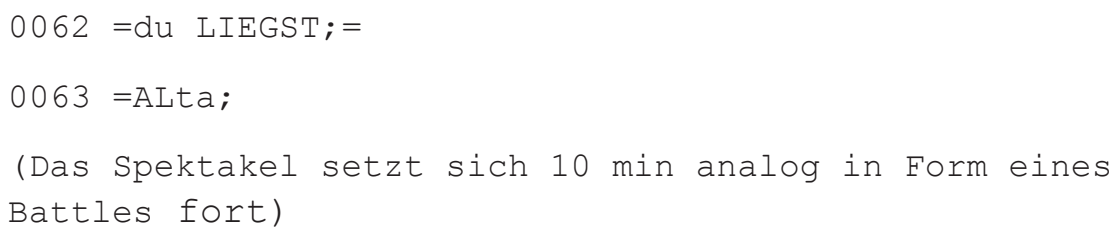

Der Ausschnitt beginnt mit der an die Jungen im Zelt adressierten Aufforderung des Betreuers Simon (Sim), „einfach ruhig zu sein“, und der im Nachfeld realisierten Questiontag „okay“, die den Turn beendet und (ggf. ungewollt) das Rederecht übergibt (Z. 0057-0058). Marcel (Mcl) ergreift das Wort, ruft - durch die erhöhte Lautstärke provozierend - ,schlaf gut“ (Z. 0059) und intensiviert durch die nachgestellte Konstruktion [PersPron 2. Pers. Sg. + wertendes Nomen] in Z. 0060 die Provokation durch die pejorative Anrede. Die prädikative Formulierung fungiert als Deiktikon, das einem oder einer Angesprochenen durch die aus Rap-Texten bekannten Kontexte der Rapper FLER und Bushido minderwertige Eigenschaften zuspricht. Es handelt sich im Goffmanschen Sinne um einen Face-Threatening Act (Goffmann, 1967) ${ }^{2}$. Philipp (Phi) solidarisiert sich kurz darauf mit seinem Zeltfreund, indem er Simon mittels der Ellipse „ein Box“ (Z. 0061) und der unverbundenen konditionalen Fortsetzung die Folgen eines Schlagabtauschs bildlich in Aussicht stellt. Das Antezedenz sowie dessen Konsequenz könnte man mit: „Wenn ich dich schlage, dann gehst du zu Boden" angemessen paraphrasieren.

Lexik und Semantik stehen hier in Einklang mit sprachlichen Stilen, die dem deutschsprachigen „Gangsta-Rap“ entnommen sind. Dieser orientiert sich im vorliegenden Fall ${ }^{3}$ an einer möglichst harten Straßenkultur, die nicht selten durch ethnische Spannungsdarstellungen negativ aufgefallen ist. Gerade dieser verbale Schlagabtausch - das „Battle“ (vgl. Labov, 1969) bzw. das gegenseitige „Dissen“ (Deppermann \& Schmidt, 2001b) - sind durch die Musik-Medien bekannte Praktiken, die nicht genuin ethnolektal markiert sind. Sie finden aber insbesondere im Genre „Gangsta-Rap“ prominent durch Rapper mit Migrationshintergrund Verbreitung. Bei diesem verbalen Schlagabtausch, der sich nach demselben Muster [PersPron 2. Pers. Sg. + wertendes Nomen] zwischen dem Betreuer und den Teilnehmern noch 10 Minuten abwechselnd fortsetzt, scheint zumindest ein qualitativer Vergleich mit den Vorbildern nicht gegeben. Das Battle in diesem Beispiel ist qualitativ zu minderwertig, als dass es mit den medialen Vorbildern ernsthaft assoziiert werden könnte. Dennoch bleibt der Versuch bestehen, die

2 Für die intergruppale Kommunikation könnte dies u.U. anders gedeutet werden, da es in Jugendgruppen durchaus gängige Praxis sein kann, sich ex negativo durch Beleidigungen auch Nähe auszusprechen. Hier beziehen sich die FTAs jedoch nicht auf ein Gruppenmitglied.

3 Vgl. die „du Kek“ Z. 0060; türkisch und albanisch für „Kuchen“. Beleidigungsformeln sind in juventulektalen Stilen oftmals mit Nahrungsmittelanreden verbunden. Z.B. „Du Lauch“. 
Muster aufzugreifen und sich eine Streetcredibility zuzulegen. Dafür sprechen die in Z. 0061f realisierte unverbundene Konstruktion „ein Box, du liegst" (vgl. u.a. Kern, 2008) sowie die markante Lexik der Szene. Über eine episodenhafte Zitation geht unser Beispiel jedoch auch nicht hinaus. Wir können wiederum feststellen, dass die ethnolektale Markierung aufgrund der nicht aktivierten weiteren Geltungsbereiche des authentischen Battles verblichen ist. Die intendierte Identitäts-Projektion liegt hier wiederum in dem Wunsch verborgen, möglichst „krass" rüberzukommen.

Unser letztes Beispiel (3) illustriert prosodische Mittel, die dem indexing Street zugehörig scheinen. In folgendem Transkript werden Charakterzüge jugendsprachlicher Erzählungen in ihrer offenen Form etabliert. Die Narration wird dabei im Rahmen einer Gruppeninteraktion initiiert, indem Tim (TIM) ab Z. 330 seine Freunde dazu auffordert, ein bestimmtes Erlebnis aus dem Berliner Freizeitbad „Insulaner“ zu erzählen. In der Geschichte geht es um Gewalterfahrungen.

$$
\text { (3) „Insulaner“ (aus Steckbauer et al., 2014) }
$$

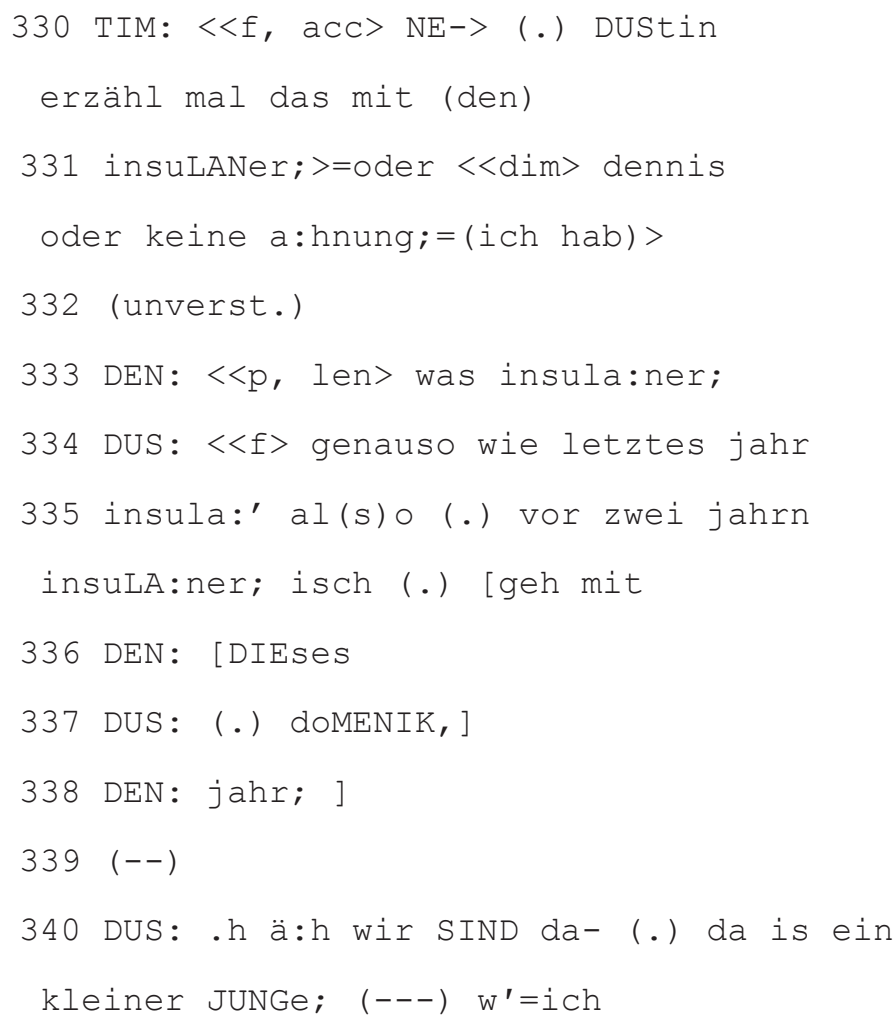




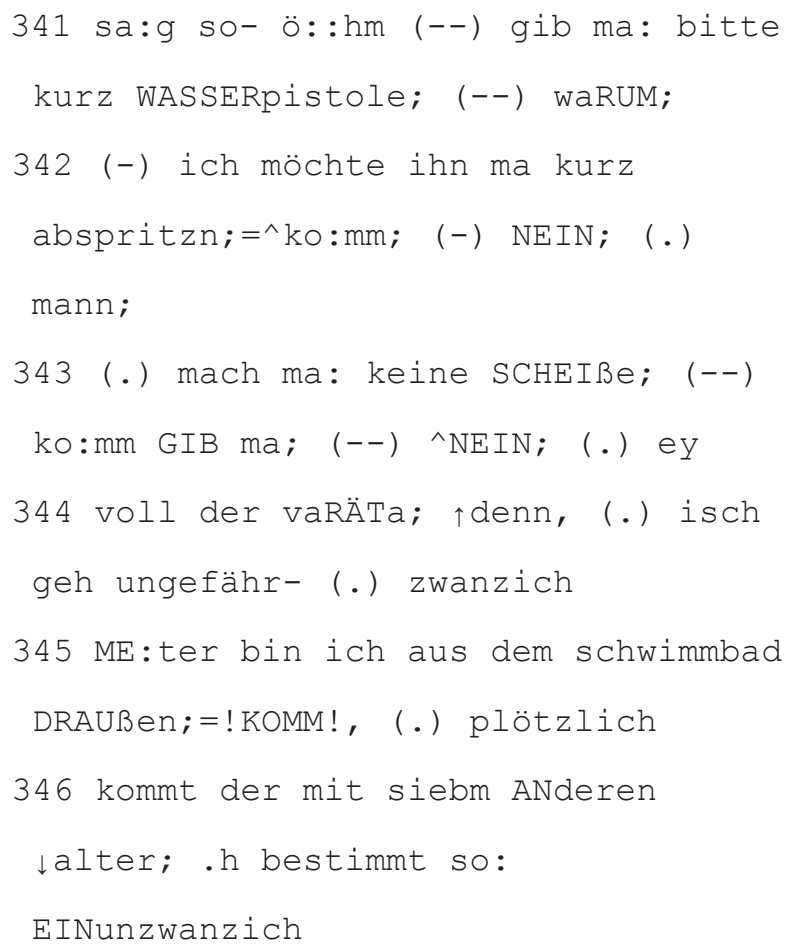

Innerhalb des Freundeskreises ist die Geschichte bekannt, was sich anhand der expliziten Referenz (Z. 330-331: „erzähl mal das mit (den) Insulaner“) deutlich wird. Sie gilt als „krasse“ Geschichte und wird immer wieder zur Wiederbelebung des gemeinsamen Erlebnisses in Interaktionen eingebracht, um Außenstehenden gegenüber die Gruppenidentität als „krasse“ Truppe zu präsentieren und den Gruppenzusammenhalt zu demonstrieren.

Dustin (DUS) kommt Tims Aufforderung nach und übernimmt selbstgewählt die Erzählerrolle. Da die Geschichte den anderen Gruppenmitgliedern bereits 
bekannt ist, etabliert sich interessanterweise ein mehr oder weniger gemeinsames Erzählen: Durch Nachfragen und Kommentare beeinflussen die anderen den Erzählverlauf (vgl. Z. 336 und 348), sodass die Erzählerrolle nicht allein bei Dustin bleibt.

In der Geschichte wird ein Erlebnis aus einem Berliner Freibad geschildert, welches grob in die Kategorie der Gewalterfahrung bzw. Konfliktsituation eingeordnet werden kann. Nach einer verbalen Auseinandersetzung mit einem Jungen mit türkischem Hintergrund, der seine Wasserpistole nicht an Dustin verleihen wollte, kommt es außerhalb des Schwimmbads zu einer verbalen Auseinandersetzung zwischen dem Sprecher (Dustin) und einer größeren Gruppe Jugendlicher, zu der auch der Bruder des Jungen gehört.

Die Geschichte bietet enormes narratives Potential, welches auf der Schilderung eines ,unglaublichen Vorfalls“ (das implizit geäußerte Unverständnis darüber, aufgrund einer Lappalie mit einer größeren Gruppe Jugendlicher in Streit zu geraten), der Art der kontrastiven Gegenüberstellung zwischen dem Jungen und Dustin sowie dem Modus des Erzählens selbst basiert. Zum einen wird die wiedergegebene Rede in besonderer Art und Weise ausgeführt. Der Sprecher Dustin springt in beide Rollen und konstruiert den stattgefundenen Dialog ganz ohne deiktische Marker. So ist nur der Beginn des Dialogs (Z. 340-341) durch das verbum dicendi sagen und einem Verzögerungssignal mit anschließender Pause markiert. Zum anderen nutzt der Sprecher im weiteren Verlauf Pausen und Prosodie, um einen lebendigen, authentischen Zugang zur erzählten Wirklichkeit zu schaffen. Er verwendet den Modus der direkten Rede (Z. 340-344), um den Dialog der Konfliktsituation wiederzugeben, wobei die Zuhörer die Aussagen den jeweiligen Sprechern selbst zuordnen müssen. Dustin markiert die unterschiedliche Zugehörigkeit der Äußerungen über paraverbale Mittel. Zudem wird der Sprachduktus des Erzähler-Ichs gegenüber dem erzählten Anderen genutzt, um die dialogische Abfolge kontrastiv zu gestalten. Die Redeanteile des erzählten Dialogpartners werden ausschließlich als Einwort-Konstruktionen wiedergegeben (Z. 341, 342, 343). Die ,erzählte Stimme“ des Erzählers ist rhythmisch hämmernd (Stakkato), dabei jedoch nicht unruhig und in einem tiefen Tonhöhenregister gehalten. So wird zum einen Coolness angezeigt (vgl. Schwitalla, 2011, S. 77-81). Zum anderen handelt es sich dabei um Merkmale ethnolektalen Sprechens junger Türken (vgl. Kern, 2008). Das Gegenüber wird prosodisch kontrastiv durch eine erhöhte Lautstärke und sog. Springtonkonstruktionen realisiert (Z. 343: ^NEIN. ; Z. 341: wa^RUM;; vgl. Selting, 2000). Den Dialog abschließend wird der kleine Junge pejorativ als „,voll der vaRÄta“ (Z. 344) betitelt.

Im weiteren Verlauf der Erlebnisschilderung fallen das mit der Zungenspitze gerollte /r/ und die Reduktion auch betonter Silben auf. Durch die Veränderung der Vokalqualität in unbetonten Silben erhalten diese mehr Gewicht, was für das Deutsche eher unüblich ist. 
Innerhalb der rhythmisch gesprochenen Äußerungen werden Präpositionen und Artikel (z.B. Z. 341) ausgelassen. Dies ist möglicherweise auf den Einfluss des Türkischen zurückzuführen, da Präpositionen und Artikel dort keine Wortklassen bilden. Allerdings handelt es sich in der Rede Dustins vermutlich eher um stilistische Mittel, denen er sich bedient, da die Auslassungen nicht durchgehend beobachtbar sind. Vereinzelt kommt es außerdem zur fehlerhaften Kasusmarkierung (Z. 350).

Auch in diesem Transkript scheinen Elemente des Kiez- und Türkendeutschen deutlich sichtbar zu sein. Wenn auch der gesamte Kontext ein multikulturelles Erlebnis beschreibt, werden stilistische Elemente, die in der Literatur oftmals Ethnolekten zugeschrieben wurden, eher nicht mit der Ethnie in Verbindung gebracht. Indiziert wird auch hier eine Straßentauglichkeit - es gilt, die Geschichte möglichst „krass“ zu erzählen.

\section{Fazit}

Unsere Analysen waren sicherlich nicht exhaustiv und die Beispiele mögen wohl eher die Befürchtungen von Ausbilder*innen, Lehrer*innen und Eltern nähren, der Jugend mangle es trotz anders ausfallender Befunde der Wissenschaft an Sprachsensibilität (vgl. dazu u.a. Efing, 2016, S. 240). Die gebotene Kürze des Beitrags hat dazu geführt, dass wir uns auf eine selektive Auswahl an Merkmalen konzentrieren mussten, die in unserem Korpus sehr prominent vertreten sind, die gleichsam auch zu einer Verzerrung der Realität führen mögen. Es ist jedoch deutlich geworden, dass Elemente, die in der Literatur dem Kiez- oder Türkendeutschen ursprünglich zugeordnet wurden, frequent auch in monolingualen Daten auftreten. Die Gründe dafür sind nicht immer offenliegend. Es mag der direkte oder indirekte interaktionale Kontakt sein, oftmals kann aber auch ein Einfluss medialer Vermittlung zur Geltung kommen. An dieser Stelle müssen auch selbstkritisch Schwächen einer rein induktiven Vorgehensweise eingeräumt werden, die ohne eine a priori Thesenbildung auskommen muss. Triangulationen von Daten, auch metasprachlicher Art, hätten hier weiterführen können.

Unsere Daten spiegeln den Wunsch der Jugendlichen, zeitweise im Hier-undjetzt eine gewisse Härte auszustrahlen, Normen der Erwachsenenwelt zu übertreten und Tabus zu brechen. Es handelt es sich bei solchen Identitäts-Positionierungen um Acts-of-Identity (Le Page \& Tabouret-Keller, 1985), die aufgrund von zugeschriebenen Merkmalen (Härte, Coolness) Stile reproduzieren. Dies gehört zur Entwicklung eines Menschen (Hurrelmann \& Quenzel, 2016). Im Zuge des stilistischen Ausprobierens und der Übernahme ursprünglich ethnolektaler Stile gehen aber bestimmte andere Merkmale (z.B. die ethnische Markierung) verloren.

Wir konnten zeigen, dass die Spiegelung einer Ethnie in unseren Daten eine nur untergeordnete oder indirekte Rolle spielt. Oftmals wurden verschiedene Ethnien und ihr Sprachgebrauch mit einer gewissen Straßentauglichkeit und 
Härte in Verbindung gebracht. Bahlo (2009) zeigt in Umfragen mit männlichen Berliner Jugendlichen, dass sie sehr bewusst türkische und arabische Einsprengsel übernehmen, um ,hart rüberzukommen“. Diesen Befund bestätigen auch Springsits und Dirim (2016) in ihrem Beitrag „Türkisch ist voll gangsta!“. Mag dies vor über 10 Jahren besonders für männliche Jugendliche gegolten haben, scheinen die Geschlechterdifferenzierungen heute zunehmend aufzuweichen, wie der zuletzt genannte Beitrag nahelegt. Auch junge Frauen orientieren sich zeitweise an harten straßentauglichen Umgangsformen.

Das Jugendsprache-im-Längsschnitt-Korpus der Jahre 2005-2010 zeigt den Beginn einer Deethnisierung, der es jedoch an Frequenz mangelt. Denkbar ist eine Verortung der gezeigten Daten im Spektrum jugendlicher Stile, die zu dem Zweck eingesetzt werden, sich möglichst hart zu zeigen und eine Straßentauglichkeit zu indizieren.

Von einer vollständigen Deethnisierung zu sprechen, scheint verfrüht. Das frequente Vorkommen, das nicht auf einzelne monolinguale Gruppen beschränkt bleibt, wird darüber entscheiden, ob sich diese Merkmale auch über einen längeren Zeitraum verfestigen und ob sie systematisch durchgängig zu beschreiben sind. Als Desiderat kann weiterhin festgehalten werden, dass eine gewisse Diskrepanz zwischen „echten“ Deethnisierungsprozessen und sozialidentifikatorischem „Zubringerfunktionen“ zu bestehen scheint, die von Folgestudien ggf. näher zu bestimmen wäre: Die Sprecher*innen mit Migrationshintergrund verwenden neben (parallel zu) ethnolektal geprägtem Deutsch in interaktiven Konstellationen - die aufgrund der formalen Situation oder des interaktiven Gebotenseins Deutsch verlangen oder nahe legen - auch eine andere Sprache (Ausgangssprache, erworbene Erstsprache, familiärer Kommunikationsmodus), die sie mit solchen Jugendlichen teilen, die den gleichen oder einen ähnlichen Hintergrund haben. In kleineren interaktiven „Neben-Situationen ${ }^{\text {ee }}$ kommt dieser (zumindest) „zweitidentitäre ${ }^{\text {ee }}$ Sprachgebrauch zur Anwendung, was unterstreicht, dass viele dieser Sprecher*innen eine kommunikative und soziale „Doppelidentitäte haben, zumindest eine kommunikative „Haupt-e und "Nebenidentitäte (vgl. Dittmar \& Simsek, 2017). Genau dies trifft aber für Sprecher*innen ohne Migrationshintergrund - auch wenn sie in der Schule gelerntes Englisch oder Französisch (u.a.) hervorragend beherrschen - nicht zu. Es fehlt die familienbezogene, lebensgeschichtliche, existenziell-schicksalhafte Rolle der Erstsprache im familiären Kontext. Um es polemischer zu sagen: die lebensweltlich-existenziell (meist in der Kindheit langfristig) erworbene zweite Sprache fällt einfach unter den Tisch. Der „deutschfixierte“ Germanistenblick spart immer wieder den auf der kommunikativen Oberfläche (oft) weniger einschlägig sichtbaren Bereich des zweisprachigen/doppelsprachigen/bi-/-tri oder multikulturellen Sprachgebrauchs aus. Soziolinguistische Differenzierungen nach Typ der Situation (z.B. Situationen, in denen „Straßenauglichkeit" demonstriert 
werden soll vs. andere Situationen, in denen „Inszenesetzen ${ }^{\text {ee }}$ und „Wettstreiten keine Rolle spielen) und der an der Interaktion Beteiligten (Status, Rollen u.a.) dürften unseres Erachtens zu graduellen Gebrauchszuschreibungen führen.

\section{References}

Androutsopoulos, J. (1998). Deutsche Jugendsprache: Untersuchungen zu ihren Strukturen und Funktionen. Frankfurt am Main: Peter Lang Verlag.

Androutsopoulos, J. (2001). From the streets to the screens and back again: on the mediated diffusion of ethnolectal patterns in contemporary German. LAUD Linguistic Agency, 522. Essen: Universität Essen.

Artamonova, O. V. (2016). Ausländersein an der Hauptschule. Bielefeld: transcript.

Auer, P. (2003). „Türkenslang“ - ein jugendsprachlicher Ethnolekt des Deutschen und seine Transformationen. In A. Häcki Buhofer (Ed.), Spracherwerb und Lebensalter (pp. 137-162). Tübingen, Basel: Francke.

Auer, P. (2016). Ethnische Marker im Deutschen zwischen Varietät und Stil. In A. Deppermann (Ed.), Das Deutsch der Migranten (pp. 9-40). Berlin, Boston: De Gruyter.

Auer, P., \& Hinskens, F. (2005). The role of interpersonal accommodation in a theory of language change. In P. Auer, F. Hinskens, \& P. Kerswill (Eds.), Dialect change. Convergence and divergence in European Languages (pp. 335-357). Cambridge: Cambridge University Press.

Bahlo, N. (2009). uallah und / oder ich schwöre. Jugendsprachliche expressive Marker auf dem Prüfstand. Gesprächsforschung - Online-Zeitschrift zur verbalen Interaktion, 11, 101-122.

Bahlo, N., Becker, T., Kalkavan-Aydın, Z., Lotze, N., Marx, K., Schwarz, C., \& Şimşek, Y. (Eds.) (2019). Jugendsprache: Eine Einführung. Wiesbaden: Springer.

Bahlo, N., \& Fladrich, M. (2016). Transkriptband Jugendsprache: Gesprochene Sprache in der Peer-Group. Berlin: Retorika.

Birkner, K., Auer, P., Bauer, A., \& Kotthoff, H. (2020). Einführung in die Konversationsanalyse. Berlin, Boston: De Gruyter. DOI:10.1515/9783110689082.

Deppermann, A. (2008). Gespräche analysieren: Eine Einführung. Wiesbaden: Verlag für Sozialwissenschaft. DOI: 10.1007/978-3-531-91973-7.

Deppermann, A., \& Schmidt, A. (2001a). Hauptsache Spaß - Zur Eigenart der Unterhaltungskultur Jugendlicher. Der Deutschunterricht, 6, 27-37.

Deppermann, A., \& Schmidt, A. (2001b). „Dissen“: Eine interaktive Praktik zur Verhandlung von Charakter und Status in Peer-Groups männlicher Jugendlicher. Osnabrücker Beiträge zur Sprachtheorie, 62, 79-98.

Dirim, I., \& Auer, P. (2004). Türkisch sprechen nicht nur die Türken. Über die Unschärfebeziehungen zwischen Sprache und Ethnie in Deutschland. Berlin, Boston: De Gruyter.

Dittmar, N., \& Şimşek, Y. (2017). Das Deutsch von Jugendlichen mit Migrationshintergrund. In (Ed.), Vielfalt und Einheit der deutschen Sprache. Zweiter Bericht zur Lage der deutschen Sprache (pp. 146-191). Tübingen: Stauffenburg.

Eckert, P., \& McConnell-Ginet, S. (1992). Think practically and look locally: Language and gender as community-based practice. Annual Review of Anthropology, 21, 461-490. DOI: 10.1146/ annurev.an.21.100192.002333.

Efing, Ch. (2016). Irgendwann muss man ja mal erwachsen werden. Spracheinstellungen und Sprach(differenz)bewusstheit in Hinblick auf Jugendsprache bei (Berufs-)SchülerInnen. In C. Spiegel, \& D. Gysin (Eds.), Jugendsprache in Schule, Medien und Alltag (pp. 239-258). Frankfurt am Main: Peter Lang Verlag.

Füglein, R. (2000). Kanak Sprak. Eine ethnolinguistische Untersuchung eines Sprachphänomens im Deutschen (Diploma thesis). Fakultät der Sprach- und Literaturwissenschaften, Universität Bamberg, Germany. 
Garfinkel, H. (1967). Studies in Ethnomethodology. Englewood Cliffs: Prentice Hall.

Goffman, E. (1967). On Face-Work. An Analysis of Ritual Elements in Social Interaction. In E. Goffmann (Ed.), Interaction Ritual (pp. 5-45). New York: Doubleday.

Grimm, W. (1986). Bericht über das Deutsche Wörterbuch (1846). In J. Grimm, \& W. Grimm (Eds.), Über das Deutsche. Schriften zur Zeit-, Rechts-, Sprach- und Literaturgeschichte (pp. 209220). Leipzig: Reclam.

Herrgen, J. (1986). Koronalisierung und Hyperkorrektion. Das palatale Allophon des /ch/-Phonems und seine Variation im Westmitteldeutschen. Stuttgart: Franz Steiner Verlag.

Hurrelmann, K., \& Quenzel, G. (2016). Lebensphase Jugend: Eine Einführung in die sozialwissenschaftliche Jugendforschung. Grundlagentexte Soziologie. Weinheim, Basel: Beltz Juventa.

Kern, F. (2008). Prosodie und Syntax im Türkendeutschen (Habilitation thesis). Philosophische Fakultät, Universität Potsdam, Germany.

Keupp H. (2012). Identität und Individualisierung: Riskante Chancen zwischen Selbstsorge und Zonen der Verwundbarkeit - sozialpsychologische Perspektiven. In H. G. Petzold (Ed.), Identität (pp. 77-105). Wiesbaden: Verlag für Sozialwissenschaften.

Labov, W. (1969). A Study of Nonstandard English. Washington DC: National Council of Teachers of English.

Le Page, R. B., \& Tabouret-Keller, A. (1985). Acts of identity: Creole-based approaches to language and ethnicity. Cambridge: Cambridge University Press.

Sacks, H., E., Schegloff, A., \& Jefferson, G. (1974). A simplest systematics for the organization of turn-taking for conversation. Language, 50(4), 696-735.

Schwitalla, J. (2011). Gesprochenes Deutsch. Eine Einführung. Berlin: Erich Schmidt Verlag.

Selting, M. (2000). Berlinische Intonationskonturen: Der „Springton“. Deutsche Sprache, 28(3), 193-231.

Selting, M., \& Couper-Kuhlen, E. (2001). Forschungsprogramm „Interaktionale Linguistik“. Linguistische Berichte, 187, 257-287.

Selting, M., Auer, P., Barth-Weingarten, D., Bergmann, J. R., Bergmann, P., Birkner, K ... Uhmann, S. (2009). Gesprächsanalytisches Transkriptionssystem 2 (GAT 2). Gesprächsforschung - Online-Zeitschrift zur verbalen Interaktion, 10, 353-402.

Springsits, B. \& Dirim, İ. (2016). „Türkisch ist voll gangster!“. In T. Geier, \& K. Zaborowski (Eds.), Migration: Auflösungen und Grenzziehung: Perspektiven einer erziehungswissenschaftlichen Migrationsforschung (pp. 135-152). Wiesbaden: Springer.

Steckbauer, D., Bahlo, N., Dittmar, N., \& Pompino-Marschall, B. (2014). „... erzähl mal das mit dem Insulaner...". Formale, funktionale und prosodische Aspekte jugendsprachlicher Narrationen. In H. Kotthoff, \& C. Mertzlufft (Eds.), Jugendsprachen: Stilisierungen, Identitäten, mediale Ressourcen (pp. 137-162). Frankfurt am Main: Peter Lang Verlag.

Thim-Mabrey, C. (2003). Sprachidentität - Identität durch Sprache. Ein Problemaufriss aus Sprachwissenschaftlicher Sicht. In N. Janich, \& C. Thim-Mabrey (Eds.), Tübinger Beiträge zur Linguistik: Vol. 465. Sprachidentität - Identität durch Sprache (pp. 1-18). Tübingen: Narr.

Wiese, H. (2012). Kiezdeutsch. Ein Dialekt entsteht. München: C.H. Beck.

Zaimoglu, F. (2011). Kanak Sprak. KiWi Paperback: Vol. 1210. Köln: Kiepenheuer \& Witsch. 